\title{
Measurement of top quark properties at CMS
}

\author{
Jónatan Piedra*† \\ Instituto de Física de Cantabria (CSIC-UC) \\ E-mail: piedraecern.ch
}

\begin{abstract}
Measurements of top quark properties in top quark decays are presented, using data collected by the CMS experiment during the years 2011 and 2012. The polarization of W bosons in top quark decays is measured. The $\mathrm{W}$ boson helicity fractions and angular asymmetries are extracted, and limits on anomalous contributions to the Wtb vertex are determined. Furthermore, searches for flavor changing neutral currents in top quark decays are presented. The flavor contents in top quark pair events are measured using the fraction of top quarks decaying into a $\mathrm{W}$ boson and $\mathrm{a} b$ quark relative to all top quark decays, $R=B R(\mathrm{t} \rightarrow \mathrm{Wb}) / B R(\mathrm{t} \rightarrow \mathrm{Wq})$, and the result is used to determine the CKM matrix element $V_{\mathrm{tb}}$ as well as the width of the top quark resonance.
\end{abstract}

The European Physical Society Conference on High Energy Physics

22-29 July 2015

Vienna, Austria

*Speaker.

${ }^{\dagger}$ For the CMS Collaboration. 


\section{The CMS detector}

The central feature of the CMS apparatus is a superconducting solenoid, which provides an axial magnetic field of 3.8 T. Within the field volume there are a silicon pixel and strip tracker, a lead tungstate crystal electromagnetic calorimeter, and a brass or scintillator hadron calorimeter. Charged-particle trajectories are measured by the tracker, covering $0 \leq \phi \leq 2 \pi$ in azimuth and $|\eta|<2.5$ in pseudorapidity, where $\eta$ is defined as $-\ln [\tan (\theta / 2)]$, and $\theta$ is the polar angle of the trajectory of the particle with respect to the counterclockwise proton beam direction. Muons are identified and measured in gas-ionization detectors embedded in the steel flux return yoke outside the solenoid. A more detailed description of CMS detector can be found in Ref. [1].

\section{W polarization}

Top quarks decay almost exclusively into a b quark and a $\mathrm{W}$ boson via the electroweak interaction. Due to its large mass, the top quark decays before hadronizing and therefore it provides the possibility to study the Lorentz structure and coupling of the Wtb vertex. In particular, the measurement of the $\mathrm{W}$ boson polarization in the top quark decays allows to probe the Wtb structure and to search for possible extentions of the standard model (SM) [2]. In general, W bosons in the top quark decays can be produced in three states of left-handed, right-handed, and longitudinal helicity. Defining $\Gamma_{L, R, 0}$ as the partial widths of the top quark decaying into left-handed, right-handed, and longitudinal $\mathrm{W}$ boson helicities, the helicity fractions are given by $F_{L, R, 0}$ and satisfy $F_{R}+F_{L}+F_{0}=1$. Including the next-to-next-to-leading-order (NNLO) QCD and electroweak corrections, the $\mathrm{W}$ boson helicity fractions are calculated to be $F_{R}=0.0017 \pm 0.0001$, $F_{0}=0.687 \pm 0.005$, and $F_{L}=0.311 \pm 0.005$ [3]. The $\mathrm{W}$ boson helicity fractions can be measured using the angular distributions of the top quark decay products. The angle $\theta_{\ell}^{*}$ is defined as the angle between the 3-momentum of the charged lepton in the $\mathrm{W}$ boson rest frame and the momentum of the $\mathrm{W}$ boson in the top quark rest frame. The angular distribution of $\cos \left(\theta_{\ell}^{*}\right)$ is parametrized as [4]:

$$
\frac{1}{\Gamma} \frac{d \Gamma}{d \cos \theta_{\ell}^{*}}=\frac{3}{8}\left(1-\cos \theta_{\ell}^{*}\right)^{2} F_{L}+\frac{3}{8}\left(1+\cos \theta_{\ell}^{*}\right)^{2} F_{R}+\frac{3}{4} \sin ^{2} \theta_{\ell}^{*} F_{0}
$$

In this analysis [5], $\mathrm{W}$ boson helicity fractions are measured from a sample of tt events with two leptons in the final state. The method adopted for this measurement is the same as the one already used in tt semileptonic [6,7] and dileptonic final states [8], and in single top quark events [9] at 7 and $8 \mathrm{TeV}$ by the CMS experiment. The analysed data sample used in this analysis corresponds to an integrated luminosity of $19.7 \mathrm{fb}^{-1}$. Events are required to contain two charged and isolated leptons with opposite sign, missing transverse energy, and at least two $b$ tagged jets. The tt system kinematics can be determined using the $\mathrm{W}$ boson and top quark mass constraints. By applying six kinematic constraints on the kinematics of the produced particles from top decays, a set of solutions for neutrino and antineutrino momenta are found. In order to extract the W boson helicity fractions, a reweighting technique is used. It considers any arbitrary configuration of the $\mathrm{W}$ boson helicity fractions and the effects of the limited experimental resolution at the same time. The $\cos \theta_{\ell}^{*}$ distribution, which is used to perform the measurement, is presented in Fig. 1 (left). The result from the fit is shown in Fig. 1 (right). The measured $\mathrm{W}$ boson helicity fractions are $F_{L}=$ 
$0.329 \pm 0.029, F_{0}=0.653 \pm 0.026$, and $F_{R}=0.018 \pm 0.027$. These results are in agreement with the SM predictions at NNLO within $2 \sigma$ uncertainties.
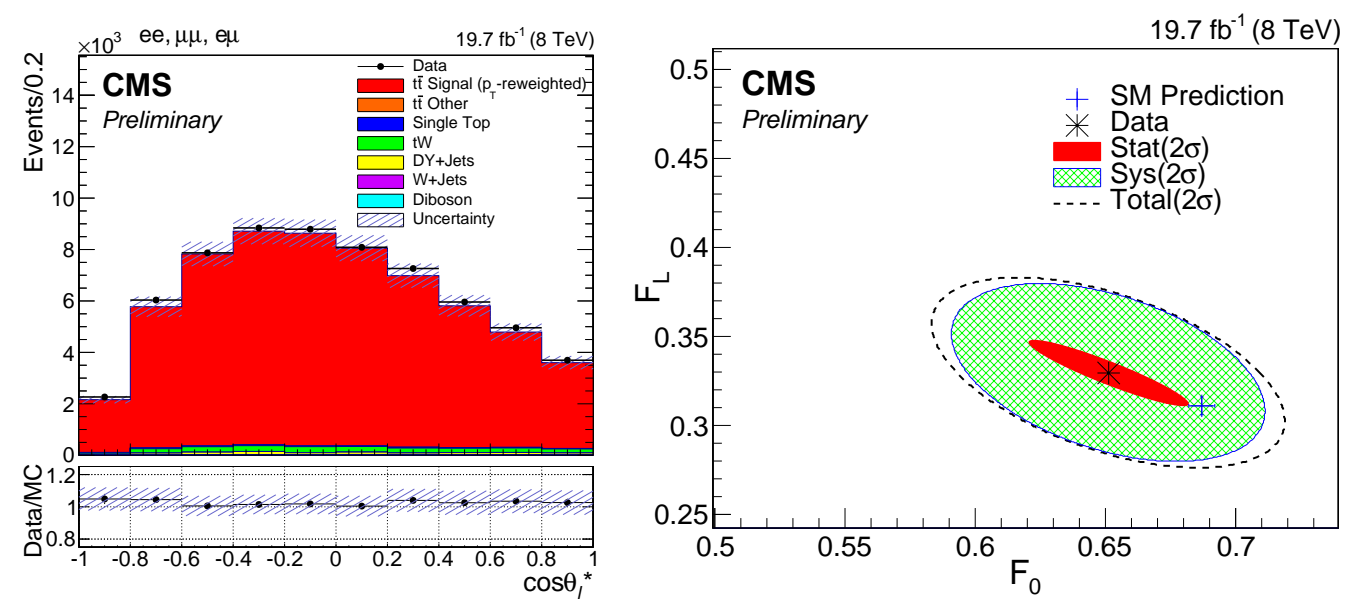

Figure 1: (Left) Distribution of $\cos \theta_{\ell}^{*}$ for the ee, $\mu \mu$, and e $\mu$ channels summed. The ratio of the number of events in data to the total number of events from simulation is shown in the lower panel. (Right) The $95 \%$ region in the $\left(F_{0}, F_{L}\right)$ plane obtained from the fit to data. The measured and theoretical values of the W boson helicity fractions are shown as well.

\section{Top quark couplings}

Within the SM, the flavor-changing neutral current (FCNC) decay of the top quark to a $\mathrm{Z}$ boson and a light up-type quark ( $\mathrm{u}$ or $\mathrm{c}$ ), is suppressed by the GIM mechanism, occurring only at the quantum loop level, with a branching fraction $\mathscr{B}(\mathrm{t} \rightarrow \mathrm{Zq})$ at $\mathscr{O}\left(10^{-14}\right)$. The detection of FCNC $\mathrm{t} \rightarrow \mathrm{Zq}$ decays at a higher-than-expected rate would thus be clear evidence for physics beyond the SM. This search [10] is performed by looking for tt events where one top quark decays into Zq and the other decays into $\mathrm{Wb}$, with both vector bosons decaying leptonically. Events with two opposite sign, same flavor, isolated leptons, consistent with a $\mathrm{Z}$ boson decay, and one extra charged lepton, are selected. Neutrinos from $\mathrm{W}$ boson decays escape detection, thus we require the missing $E_{T}$ to be larger than $30 \mathrm{GeV}$. To reduce the background from diboson events we require at least two jets, and exactly one of these jets should be tagged as a b quark jet. The invariant mass of the $\mathrm{W}$ boson and the $\mathrm{b}$ tagged jet, $m_{\mathrm{Wb}}$, is required to be within $35 \mathrm{GeV}$ of the top quark mass. A non-b jet is combined with the $\mathrm{Z}$ candidate to form a second top quark candidate. By examining all possible pairings, the top quark candidate which has the largest separation in azimuthal angle to the first top quark is selected, and the reconstructed top quark mass, $m_{\mathrm{Zj}}$, is required to be within $25 \mathrm{GeV}$ of the assumed value of $172.5 \mathrm{GeV}$. Fig. 2 shows the comparison of the $m_{\mathrm{Zj}}$ and $m_{\mathrm{Wb}}$ distributions in data and simulation. After applying all the criteria 3.1 \pm 1.1 events are expected from SM background processes and 1 event is observed in data. A 95\% C.L. upper limit on the branching fraction of the $\mathrm{t} \rightarrow \mathrm{Zq}$ decay is determined using the modified frequentist approach. Combining this result with a previous search corresponding to an integrated luminosity of $5 \mathrm{fb}^{-1}$ at $\sqrt{s}=7 \mathrm{TeV}$, excludes a $\mathrm{t} \rightarrow \mathrm{Zq}$ branching fraction greater than $0.05 \%$ at a confidence level of $95 \%$. 

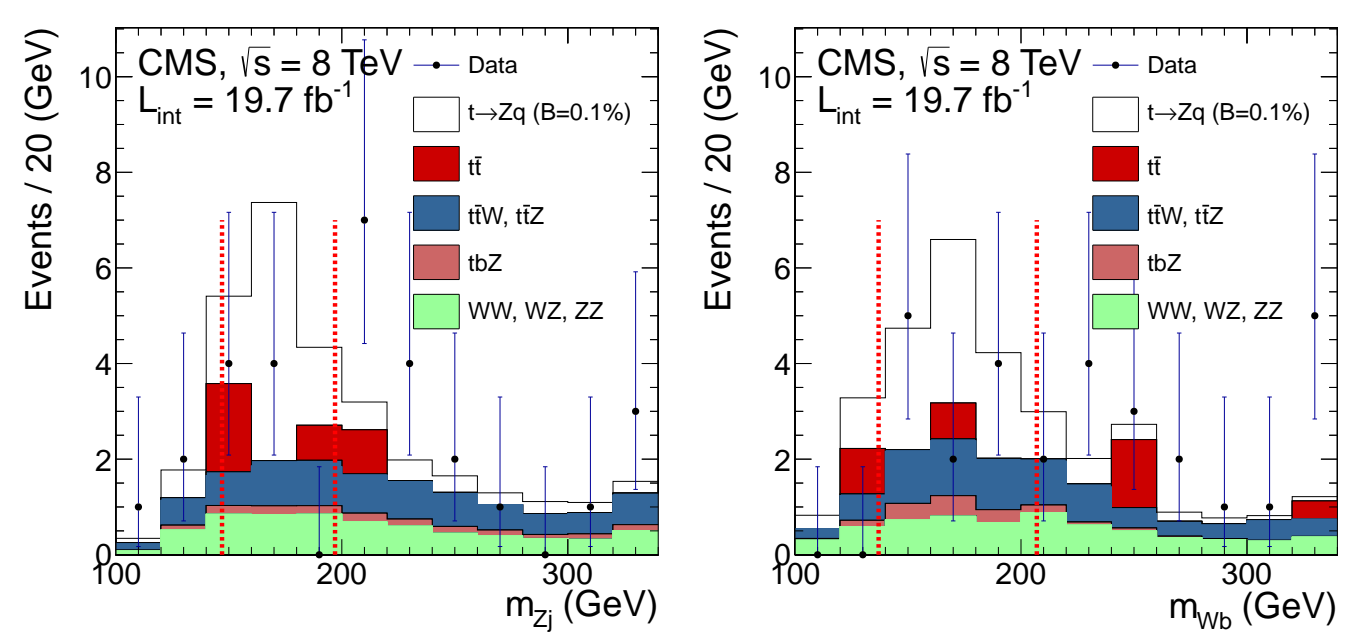

Figure 2: Comparison between data and simulated events of the $m_{\mathrm{Zj}}$ (left) and $m_{\mathrm{Wb}}$ (right) distributions after the event selection prior to the top quark mass requirements, which are shown as the dotted vertical lines. The data are represented by the points with error bars and the open histogram is the expected signal. The stacked solid histograms represent the dominant backgrounds. The last bin in each of the two plots contains all the overflow events.

\section{Top quark pair production in association with a $\mathrm{W}$ or $\mathrm{Z}$ boson}

The $t \mathrm{tZ}$ production cross section provides the most accessible direct measurement of the top quark coupling to the $\mathrm{Z}$ boson. Both $\sigma(\mathrm{ttW})$ and $\sigma(\mathrm{ttZ})$ would be altered in a variety of new physics models that can be parameterized by dimension-six operators added to the standard model Lagrangian. We present [11] the first measurements of the ttW and $t \mathrm{Z}$ cross sections using full event reconstruction techniques. We target events in which the associated $\mathrm{W}$ boson decays to a charged lepton and a neutrino or the $\mathrm{Z}$ boson decays to two charged leptons. Events for this analysis are divided into five mutually exclusive channels. The opposite-sign dilepton channel targets $\mathrm{ttZ}$ events where the $\mathrm{Z}$ boson decays into an opposite sign pair of electrons or muons, and the $t$ system decays hadronically. The same sign dilepton channel selects ttW events in which the associated $\mathrm{W}$ boson, and the $\mathrm{W}$ boson of the same charge from the tt system, each decay to a lepton and a neutrino, and the remaining $\mathrm{W}$ boson decays to quarks. The three lepton $\mathrm{ttW}$ channel targets events in which both the associated $\mathrm{W}$ and the pair of $\mathrm{W}$ bosons from the tt pair decay leptonically. Furthermore, no same flavor opposite sign pair of leptons can have a mass within $10 \mathrm{GeV}$ of the $\mathrm{Z}$ boson mass. The three lepton $\mathrm{tt} Z$ channel selects events in which the $\mathrm{Z}$ boson decays to a pair of electrons or muons, and one $\mathrm{W}$ boson from the tt system decays to a charged lepton and a neutrino, with the remaining $\mathrm{W}$ boson decaying to quarks. The selection is identical to the three lepton ttW channel, except that at least one same flavor opposite sign pair of leptons must have an invariant mass within $10 \mathrm{GeV}$ of the $\mathrm{Z}$ boson mass. Events with four leptons come from ttZ decays in which the $\mathrm{Z}$ boson and both $\mathrm{W}$ bosons from the tt decay produce electrons or muons.

In each channel we attempt a full or partial reconstruction of the ttW or ttZ system with a linear discriminant that matches leptons and jets to their parent particles using mass, charge, and $\mathrm{b}$ tagging information. Additional kinematic variables from leptons and jets are combined with 
output from the linear discriminant in a multivariate analysis that is used to make the final measurement of the $\mathrm{ttW}$ and $\mathrm{ttZ}$ cross sections. We perform separate one-dimensional fits for the $\mathrm{ttW}$ and $\mathrm{ttZ}$ cross sections using the relevant channels for each process. The fit for each cross section is performed with the other cross section set to the standard model (SM) value and uncertainty from theory. The combined ttW cross section measurement in same sign and three lepton events is $\sigma(\mathrm{ttW})=382_{-102}^{+117} \mathrm{fb}$, corresponding to a $4.8 \sigma$ deviation from the background-only hypothesis, where a significance of $3.5 \sigma$ was expected in the standard model. Combining opposite sign, three lepton, and four lepton channels, the $\mathrm{ttZ}$ cross section is measured to be $\sigma(\mathrm{ttZ})=242_{-55}^{+65} \mathrm{fb}$, an observation with a significance of $6.4 \sigma$ from the background-only hypothesis and in agreement with the standard model expectation.

Direct measurement of the $\mathrm{ttZ}$ and $\mathrm{ttW}$ cross sections can be applied to searches for new physics (NP) within the framework of an effective field theory. The effects of new particles or interactions can be captured in a model-independent way by supplementing the SM Lagrangian with higher dimensional operators involving SM fields. To study the effects of NP on the ttW and $\mathrm{ttZ}$ processes, we compute cross sections as a function of the Wilson coefficients. Cross sections were computed for the production of $\mathrm{tt}$, a Higgs boson, $\mathrm{ttZ}$, and $\mathrm{ttW}$, sampling 20 points for each Wilson coefficient. For each sampled point, all other Wilson coefficients were fixed at zero. From this survey, we select five operators as of particular interest because they have a small effect on inclusive Higgs boson and tt production, and a large effect on $\mathrm{ttZ}$, $\mathrm{ttW}$, or both. We have placed limits on these five dimension-six operators: $\bar{c}_{u B}, \bar{c}_{H Q}^{\prime}, \bar{c}_{H Q}, \bar{c}_{H u}$, and $\bar{c}_{3 W}$. Two of them are shown, for illustration, in Fig. 3. All of the measured values are compatible with the standard model predictions, within uncertainties.
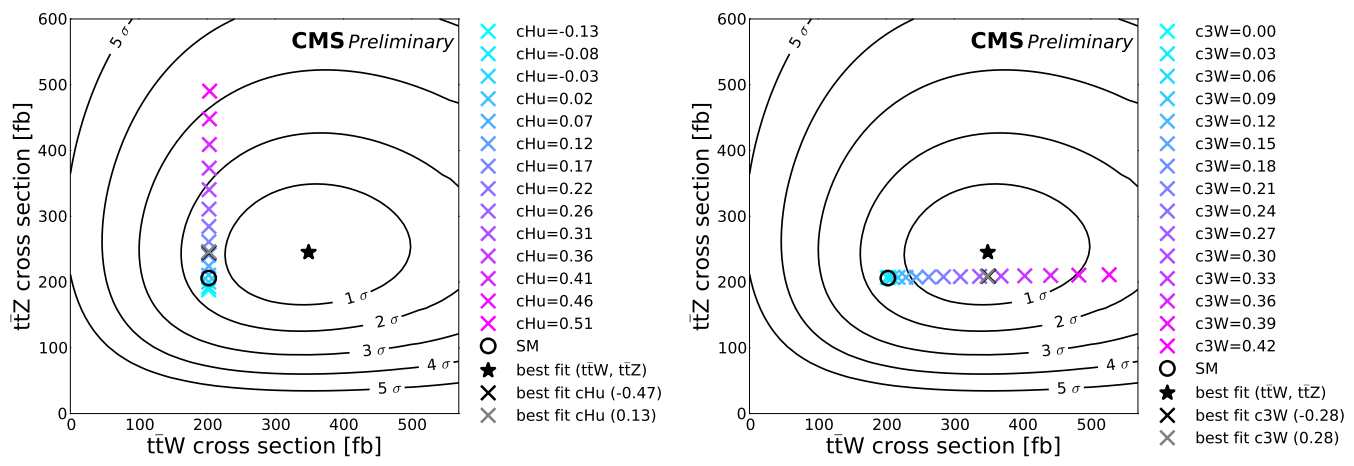

Figure 3: Sampled coefficient values for $\bar{c}_{H u}$ (left) and $\bar{c}_{3 W}$ (right), plotted in the $\sigma(\overline{\mathrm{t}} \mathrm{W}), \sigma(\mathrm{t} \bar{Z})$ plane. There are typically two best fit values, one greater and one less than zero, which lie on top of one another in the plane.

\section{Measurement of the ratio $B(\mathrm{t} \rightarrow \mathrm{Wb}) / B(\mathrm{t} \rightarrow \mathrm{Wq})$ in pp collisions at $\sqrt{s}=8 \mathrm{TeV}$}

The magnitude of the top-bottom charged current is proportional to $\left|V_{\mathrm{tb}}\right|$, an element of the Cabibbo-Kobayashi-Maskawa (CKM) matrix. It is expected to be close to unity and dominate over the off-diagonal elements. Thus, the decay modes of the top quark to lighter down-type quarks (d or s) are allowed, but highly suppressed. The indirect measurement of $\left|V_{\mathrm{tb}}\right|$, from the unitarity 
constraint of the CKM matrix, is $\left|V_{\mathrm{tb}}\right|=0.999146_{-0.000046}^{+0.000021}$. Any deviation from this value or in the partial decay width of the top quark to $b$ quarks, would indicate new physics contributions such as those from new heavy up- and/or down-type quarks or a charged Higgs boson, amongst others. Here [12] we present a measurement of $R=B(\rightarrow \mathrm{Wb}) / B(\mathrm{t} \rightarrow \mathrm{Wq})$, where the denominator includes the sum over the branching fractions of the top quark to a $\mathrm{W}$ boson and a down-type quark ( $\mathrm{q}=\mathrm{b}, \mathrm{s}, \mathrm{d})$. Under the assumption of the unitarity of the $3 \times 3 \mathrm{CKM}$ matrix, $R=\left|V_{\mathrm{tb}}\right|^{2}$, and thus to indirectly measure $\left|V_{\mathrm{tb}}\right|$. In addition, the combination of a determination of $R$ and a measurement of the t-channel single-top cross section can provide an indirect measurement of the top quark width $\Gamma_{\mathrm{t}}$.

The event selection is optimised for tt dilepton final states that contain two isolated oppositely charged leptons, missing transverse energy, and at least two jets. The selected events are categorised by the dilepton channel and the number of observed jets. The tt dilepton signal strength, $\mu$, defined as the ratio of the observed to the expected signal rate, is measured from the jet multiplicity distribution by using a profile likelihood method. A fit including all categories gives the range $0.909<\mu<1.043$ at the $68 \%$ confidence level (CL).

The $b$ flavour content of the selected events (both signal and background) is determined from the $b$ tagged jet multiplicity distribution. Fig. 4 (left) shows the number of $b$ tagged jets in the selected dilepton data sample, compared to the expectations from simulation. The multiplicity is shown separately for each dilepton channel and jet multiplicity. The expected event yields are corrected after the fit for the signal strength. For a given number of correctly reconstructed and selected jets, the expected $\mathrm{b}$ tagged jet multiplicity can be modelled as a function of $R$ and the $\mathrm{b}$ tagging and misidentification efficiencies. In the parameterisation, we distinguish events containing jets from 0,1 , or 2 top quark decays. Fig. 4 (right) shows the results obtained by maximising the profile likelihood. The combined measurement of $R$ gives $R=1.014 \pm 0.003$ (stat.) \pm 0.032 (syst.), in good agreement with the SM prediction. The largest contribution to the systematic uncertainty is from the b-tagging efficiency measurement.

A lower limit of $R>0.955$ at $95 \% \mathrm{CL}$ is obtained after requiring $R \leq 1$ and taking into account both statistical and systematical uncertainties. This result translates into a lower limit $\left|V_{\mathrm{tb}}\right|>0.975$ at $95 \% \mathrm{CL}$ when assuming the unitarity of the three-generation CKM matrix. By combining this result with a previous CMS measurement of the t-channel production cross section for single top quarks, an indirect measurement of the top quark total decay width $\Gamma_{\mathrm{t}}=1.36 \pm$ 0.02 (stat. $)_{-0.11}^{+0.14}$ (syst.) GeV is obtained, in agreement with the SM expectation. These measurements of $R$ and $\Gamma_{\mathrm{t}}$ are the most precise to date and the first obtained at the LHC.

\section{References}

[1] CMS Collaboration, JINST 3, S08004 (2008).

[2] J. A. Aguilar-Saavedra et al.,"Probing anomalous Wtb couplings in top pair decays“, Eur. Phys. J. C 50 (2007) 519-533, doi:10.1140/epjc/s10052-007-0289-4.

[3] A. Czarnecki, J. G. Korner, and J. H. Piclum, "Helicity fractions of W bosons from top quark decays at NNLO in QCD“, Phys. Rev. D 81 (2010) 111503, doi: 10.1103 /PhysRevD . 81.111503.

[4] R. Dalitz and G. R. Goldstein, "Decay and polarization properties of the top quark“, Phys. Rev. D 45 (1992) 1531, doi:10.1103/PhysRevD.45.1531. 

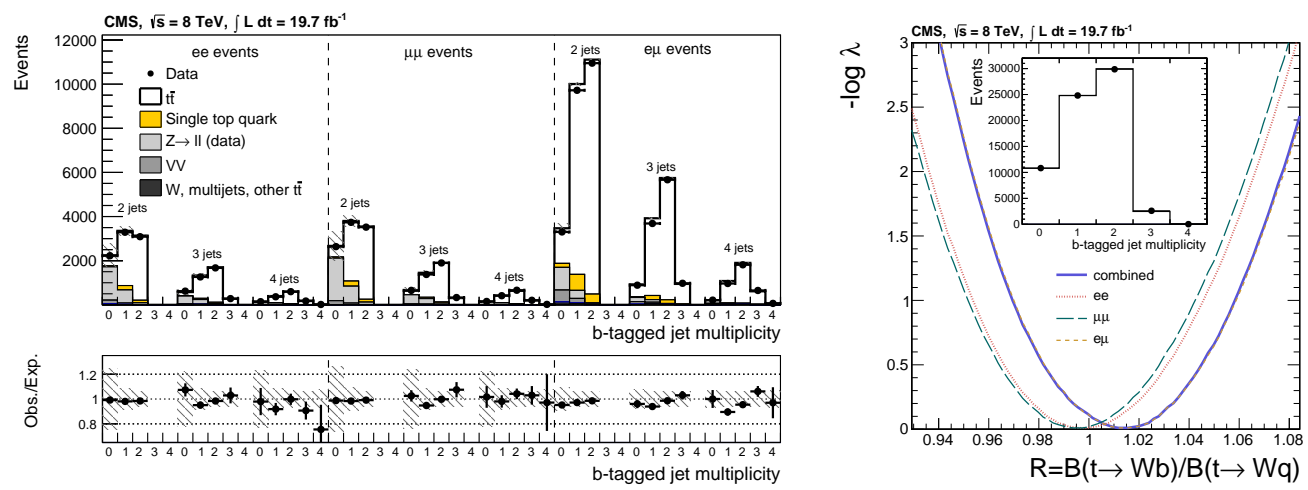

Figure 4: (Left) Number of $\mathrm{b}$ tagged jets per event for the different dilepton channels. For each final state, separate subsets are shown corresponding to events with two, three, or four jets. (Right) Variation of the log of the profile likelihood ratio used to extract $R$ from the data. The variations observed in the combined fit and in the exclusive ee, $\mu \mu$, and e $\mu$ channels, are shown. The inset shows the inclusive $\mathrm{b}$ tagged jet multiplicity distribution and the fit distribution.

[5] CMS Collaboration, "Measurement of the $\mathrm{W}$ boson helicity using ttbar events in the dilepton final state at $\sqrt{s}=8 \mathrm{TeV}$ “, CMS Physics Analysis Summary CMS-PAS-TOP-14-017, 2015.

[6] CMS Collaboration, "Measurement of the W-boson helicity in top-quark decays from tt production in lepton+jets events in pp collisions at $\sqrt{s}=7 \mathrm{TeV}$ “, JHEP 10 (2013) 167, doi:10.1007/JHEP10 (2013) 167 .

[7] CMS Collaboration, "Measurement of the W-boson helicity in top decays from tt production in lepton+jets events at the LHC at $\sqrt{s}=8 \mathrm{TeV}$ “, CMS Physics Analysis Summary CMS-PAS-TOP-13-008, 2013.

[8] CMS Collaboration, "Measurement of W-polarization in dileptonic tt events in pp collisions with $\sqrt{s}=7$ TeV“, CMS Physics Analysis Summary CMS-PAS-TOP-12-015, 2012.

[9] CMS Collaboration, "Measurement of the $\mathrm{W}$ boson helicity in events with a single reconstructed top quark in pp collisions at $\sqrt{s}=8 \mathrm{TeV}$ “, JHEP 01 (2015) 053, doi:10.1007/JHEP 01 (2015) 053.

[10] CMS Collaboration, "Search for Flavor-Changing Neutral Currents in Top-Quark Decays $\mathrm{t} \rightarrow \mathrm{Zq}$ in pp Collisions at $\sqrt{s}=8 \mathrm{TeV}$ “, PRL 112, 171802 (2014).

[11] CMS Collaboration, "Measurement of top quark pairs produced in association with a $\mathrm{W}$ or $\mathrm{Z}$ boson in pp collisions at $\sqrt{s}=8 \mathrm{TeV}$ using event reconstruction techniques", CMS Physics Analysis Summary CMS-PAS-TOP-14-017, 2015.

[12] CMS Collaboration, "Measurement of the ratio $B(\rightarrow \mathrm{Wb}) / B(\mathrm{t} \rightarrow \mathrm{Wq})$ in pp collisions at $\sqrt{s}=8$ TeV“, Physics Letter B 736 (2014) 33-57. 